\title{
Bacterial Diversity of Mangrove Soil in Karankadu from East Coast of Tamil Nadu, India
}

\author{
A. Saseeswari*, G. Kanimozhi and A. Panneerselvam \\ PG and Research Department of Botany and Microbiology, A.V.V.M. Sri Pushpam College, \\ (Autonomous) Poondi - 613 503, Thanjavur (Dt), Tamil Nadu, India \\ *Corresponding author
}

\section{Keywords}

Bacterial diversity, Mangrove soil, Morphological and Biochemical characterization.

\section{Article Info}

Accepted:

22 March 2016

Available Online:

10 April 2016

\section{A B S T R A C T}

In the present investigation about bacterial diversity from mangrove soil of Tamilnadu in four seasons were analyzed. Bacterial isolation was done by the soil dilution method incubated at $37^{\circ} \mathrm{C}$ for 24 hours. Totally twentyseven different bacterial colonies were isolated from Karankadu mangrove soil. The maximum bacterial colonies were presented in summer seasons were compare with pre-monsoon, monsoon and post-monsoon seasons. The predominant bacterial genera namely Micrococcus spp., Bacillus spp., Acetobacter spp., Pseudomonas spp., Streptococcus spp., Staphylococcus spp., Enterococcus spp., Sulfidobacillus spp., Escherichia coli., Aeromonas spp., Brevibacterium spp., Listeria spp., Azotobacter spp., Cellulomonas spp., Corynebacterium spp., Aerococcus spp., Klebsiella spp., Marinococcus spp., Enterobacter spp., Thiobacillus spp., Planococcus spp and Shigella spp.

\section{Introduction}

Mangrove forests are distributed throughout the tropical and subtropical coasts of the world. They are particularly well developed in estuarine areas of the tropics, where they reach their greatest areal extent. The World's mangroves span over 30 countries with a total area of 99,300 sq. $\mathrm{km}$. The largest mangrove area occurs in Indonesia (30\%), Brazil (10\%), Australia (8\%), India and Nigeria (7\%, each). World-wide mangroves are disappearing at an alarming rate. In some developing countries about $80 \%$ of mangroves were lost in the last three decades. Mangrove environments are unique atmosphere, harboring diverse groups of microorganisms which perform an important role in nutrient cycling and regulate chemical environment of the ecosystem (Alongi et al., 1993; Holguin et al., 1999). The free living bacteria, fungi and yeasts were reported to have significant role in formation of accrual in the mangrove ecosystems (Maria and Sridhar, 2002). The phylogenetic and functional description of microbial diversity in the mangrove ecosystem has not been well addressed to the same extent as that of the other 
environments (Zhou et al., 2006). The microbial diversity and distribution in a mangrove would improve our understanding of bacterial functionality and their interactions found in that ecosystem (Kathiresan and Selvam, 2006). Hence, in the present study investigated to assess the bacterial diversity from mangrove soil of Tamilnadu in different seasons to understand the structural and functional dynamics in the mangrove ecosystem and to identify some biotechnologically important bacteria for their further application.

\section{Materials and Methods}

\section{Sampling Schedule}

Soil samples were collected from karankadu mangrove soil in four seasons for a period of 2013-2014.

\section{Bacterial Analysis}

Soil samples were processed for isolation of bacteria. One gram sample was used for each inoculums. Serially diluted inoculums $\left(10^{-4}\right.$ to $\left.10^{-6}\right)$ were used for inoculums in pore-plating techniques for isolation of bacteria.

\section{Isolation of Pure Culture and Identification}

The colonies growing on nutrient agar plates with different morphology were counted separately. The different bacterial colonies from the mother culture were picked up by sterile inoculation loop and aseptically inoculated into the separate nutrient agar plates. These plates were incubated at $37 \pm 2^{\circ} \mathrm{C}$ for $24 \mathrm{hrs}$ and each plate contain single kind of bacteria. The isolated bacteria strains were identified based on their cultural morphological and biochemical characteristics (Cappuccino and Sherman, 1999).

\section{Results and Discussion}

The bacterial diversity of mangrove soil in different seasons results were studied (table $-1)$. Totally twenty-seven different bacterial colonies were isolated in various seasons. The maximum bacterial colonies were presented in summer seasons pre-monsoon, monsoon and post-monsoon seasons. The importance of bacterial generated detritus in mangrove areas that acts as the major substrate for bacterial growth in mangrove ecosystems was outlined in a conceptual model by Bano and Nisa (1997).

The morphologically, isolated bacterial colonies were observed round, oval, translucent, irregular and the colour was also indicated as orange, yellow, white, pink (Table-2).

The study of marine bacterial diversity is important in order to understand the community structure and pattern of distribution. Bacteria are generally less than $1-2 \mu \mathrm{m}$ in size except for the largest bacterium Epulopiscium fishlesoni, a Grampositive species $200-800 \mu \mathrm{m}$ long that lives in the gut of a Red Sea fish (Fell et al., 1984). In the marine environment, $90 \%$ of bacteria are Gram-negative with different characteristics (Zobell, 1946) and the Gramnegative cell wall is better adapted for survival in the marine environment but contradictory utmost of bacteria Grampositive in mangrove soil. Eighteen bacterial flora isolates that metabolize waste drilling fluid were collected from a mangrove swamp in Nigeria (Oliver, 1982).

The bacterial strains are identified an observational and biochemical approach has been used (Table-3). Interestingly, four additional bacterial strains isolated from the same swamp depress growth rates of Staphylococcus and Pseudomonas species and could, therefore, decrease normal rates 
of organic decomposition (Ganesh Babu et al., 2004). Similarly same isolates were observed from mangrove soil in different seasons. Certain bacterial strains such as Pseudomonas mesophilica, P. caryophylls and Bacillus cereus exhibit magnetic behavior which may be called magnetobacteria isolated from mangrove sediments of Pichavaram, Southeast India (Bernard et al., 2000).

In the present study totally twenty-seven bacterial species were identified such as Micrococcus spp., Bacillus spp.,
Acetobacter spp., Pseudomonas spp., Streptococcus spp., Staphylococcus spp., Enterococcus spp., Sulfobacillus spp., Escherichia coli., Aeromonas spp., Brevibacterium spp., Listeria spp., Azotobacter spp., Cellulomonas spp., Corynebacterium spp., Terrabacter spp., Aerococcus spp., Klebsiella spp., Marinococcus spp., Sacchrococcus spp., Enterobacter spp., Thiobacillus spp., Planococcus spp., Shigella spp., Dermobacter spp., Salinococcus spp and Deinococcus spp.(Table-3)

Table1. Number of isolated bacterial colonies present in four season of year (2013-2014)

\begin{tabular}{|l|l|l|l|l|}
\hline List of Organisms Name & Summer & Premonsoon & Monsoon & Postmonsoon \\
\hline 1. Micrococcus $\mathrm{spp}$ & + & + & + & + \\
\hline 2. Bacillus $\mathrm{spp}$ & + & + & + & + \\
\hline 3. Acetobacter $\mathrm{spp}$ & - & + & + & - \\
\hline 4. Pseudomonas $\mathrm{spp}$ & + & + & + & + \\
\hline 5. Streptococcus $\mathrm{spp}$ & + & + & + & - \\
\hline 6. Staphylococcus $\mathrm{spp}$ & + & + & + & + \\
\hline 7. Enterococcus $\mathrm{spp}$ & - & + & - & - \\
\hline 8. Sulfobacillus $\mathrm{spp}$ & + & - & + & + \\
\hline 9. Escherichia coli & + & + & + & + \\
\hline 10. Aeromonas $\mathrm{spp}$ & + & - & - & + \\
\hline 11. Brevibacterium $\mathrm{spp}$ & - & + & - & - \\
\hline 12. Listeria spp & - & - & + & - \\
\hline 13. Azotobacter $\mathrm{spp}$ & + & - & - & + \\
\hline 14. Cellulomonas $\mathrm{spp}$ & - & + & - & - \\
\hline 15. Corynebacterium $\mathrm{spp}$ & + & + & - & + \\
\hline 16. Terrabacter $\mathrm{spp}$ & + & + & - & - \\
\hline 17. Aerococcus $\mathrm{spp}$ & + & + & + & + \\
\hline 18. Klebsiella $\mathrm{spp}$ & + & + & + & + \\
\hline 19. Marinococcus $\mathrm{spp}$ & + & - & + & - \\
\hline 20. Sacchrococcus $\mathrm{spp}$ & + & + & + & + \\
\hline 21. Enterobacter $\mathrm{spp}$ & + & + & + & + \\
\hline 22. Thiobacillus $\mathrm{spp}$ & + & - & - & - \\
\hline 23. Planococcus $\mathrm{spp}$ & - & + & + & + \\
\hline 24. Shigella spp & + & - & - & - \\
\hline 25. Dermobacter $\mathrm{spp}$ & - & - & + & - \\
\hline 26. Salinococcus $\mathrm{spp}$ & + & - & - & - \\
\hline 27. Deinococcus $\mathrm{spp}$ & - & - & + & - \\
\hline+ indicates presence; - indicates absence & & & & \\
\hline
\end{tabular}


Table.2 Morphological Characterisation of isolated bacteria

\begin{tabular}{|c|c|c|c|c|}
\hline $\begin{array}{l}\text { S. } \\
\text { No. }\end{array}$ & $\begin{array}{l}\text { List of Organism } \\
\text { Name }\end{array}$ & $\begin{array}{l}\text { Morphology and Nature } \\
\text { of the colony }\end{array}$ & $\begin{array}{l}\text { Colour } \\
\text { colony }\end{array}$ & $\begin{array}{l}\text { Size of } \\
\text { colony }(\mathrm{mm})\end{array}$ \\
\hline 1 & Micrococcus spp & Convex,circular & Yellow & $0.5-1.5 \mathrm{~mm}$ \\
\hline 2 & Bacillus spp & Flat and irregular & Whitish & $1.2 \mathrm{~mm}$ \\
\hline 3 & Acetobacter spp & Circular & Pale gray & $1-3 \mathrm{~mm}$ \\
\hline 4 & $\begin{array}{l}\text { Pseudomonas } \\
\text { aeruoginosa }\end{array}$ & Round & $\begin{array}{l}\text { Diffusible } \\
\text { Green }\end{array}$ & $1.5-3 \mathrm{~mm}$ \\
\hline 5 & Streptococcus spp & Oval,translucent & White & $0.5-0.8 \mathrm{~mm}$ \\
\hline 6 & Staphylococcus spp & Convex,circular & Yellow & $1-1.8 \mathrm{~mm}$ \\
\hline 7 & Enterococcus spp & Regular & Yellow/white & $0.8 \mathrm{~mm}$ \\
\hline 8 & Sulfobacillus spp & Oval & White & $0.5-1 \mathrm{~mm}$ \\
\hline 9 & Escherichia coli & Round & Cream white & $0.5-1.0 \mathrm{~mm}$ \\
\hline 10 & Aeromonas spp & Convex,opaque & Brown & $0.8 \mathrm{~mm}$ \\
\hline 11 & Brevibacterium spp & Convex,opaque & Gray-white & $2 \mathrm{~mm}$ \\
\hline 12 & Listeria spp & Round,translucent & White & $1-2 \mathrm{~mm}$ \\
\hline 13 & Azotobacter spp & Oval & White & $1-2 \mathrm{~mm}$ \\
\hline 14 & Cellulomonas spp & Regular & Yellow/white & $1-2 \mathrm{~mm}$ \\
\hline 15 & $\begin{array}{l}\text { Corynebacterium } \\
\text { spp }\end{array}$ & Opaque,translucent & Purple & $0.5-1 \mathrm{~mm}$ \\
\hline 16 & Terrabacter spp & Round & $\begin{array}{l}\text { Gray-white to } \\
\text { yellow }\end{array}$ & $1-2 \mathrm{~mm}$ \\
\hline 17 & Aerococcus spp & Irregular & Dull white & $0.5-1 \mathrm{~mm}$ \\
\hline 18 & Klebsilla spp & Round & Grayish White & $1-2 \mathrm{~mm}$ \\
\hline 19 & Marinococcus spp & Irregular & Yellow/orange & $0.1-0.5 \mathrm{~mm}$ \\
\hline 20 & Sacchrococcus spp & Irregular & White & $0.5-1.5 \mathrm{~mm}$ \\
\hline 21 & $\begin{array}{l}\text { Enterobacter } \\
\text { aerogenes }\end{array}$ & Convex & Yellow & $0.5-1 \mathrm{~mm}$ \\
\hline 22 & Thiobacillus spp & circular & White & $1-3 \mathrm{~mm}$ \\
\hline 23 & Planococcus spp & circular & Orange & $1-2 \mathrm{~mm}$ \\
\hline 24 & Shigella spp & Circular,translucent & White & $0.5-1 \mathrm{~mm}$ \\
\hline 25 & Dermobacter spp & Circular & Greyish white & $0.5-1 \mathrm{~mm}$ \\
\hline 26 & Salinococcus spp & Circular & Orange & $0.8-1.2 \mathrm{~mm}$ \\
\hline 27 & Deinococcus spp & Regular & Red to pink & $0.3-1.5 \mathrm{~mm}$ \\
\hline
\end{tabular}


Table.3 Bio chemical Characterization

\begin{tabular}{|c|c|c|c|c|c|c|c|c|c|c|c|c|c|c|c|}
\hline \multirow{2}{*}{$\begin{array}{l}\text { S. } \\
\text { No }\end{array}$} & \multirow{2}{*}{ List of Organisms } & \multirow{2}{*}{$\begin{array}{l}\text { Gram } \\
\text { Staining }\end{array}$} & \multirow{2}{*}{ Motility } & \multirow[b]{2}{*}{ 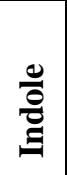 } & \multirow[b]{2}{*}{$\stackrel{s}{\Sigma}$} & \multirow[b]{2}{*}{ 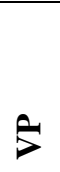 } & \multirow{2}{*}{ 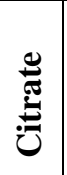 } & \multirow{2}{*}{ 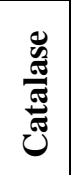 } & \multirow{2}{*}{ 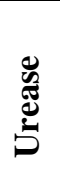 } & \multirow[b]{2}{*}{$\overline{2}$} & \multirow{2}{*}{ 旁 } & \multirow{2}{*}{ 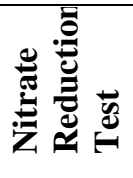 } & \multicolumn{3}{|c|}{$\begin{array}{l}\text { Carbohydrates } \\
\text { Fermentation Test }\end{array}$} \\
\hline & & & & & & & & & & & & & $\mathbf{G}$ & $\mathbf{L}$ & $\mathbf{S}$ \\
\hline 1. & Micrococcus spp & $\begin{array}{l}+\mathrm{ve} \\
\text { cocci }\end{array}$ & $\begin{array}{l}\text { Non- } \\
\text { Motile }\end{array}$ & + & - & + & - & + & + & $\begin{array}{l}\text { Alkaline } \\
\text { production }\end{array}$ & - & - & - & - & + \\
\hline 2. & Bacillus spp & +ve Rod & Motile & - & + & + & + & + & - & $\begin{array}{l}\text { Alkaline, } G \\
\text { as } \\
\text { production }\end{array}$ & + & - & + & + & \\
\hline 3. & Acetobacter spp & -ve Rod & $\begin{array}{l}\text { Non- } \\
\text { Motile } \\
\end{array}$ & - & - & - & - & + & - & $\begin{array}{l}\text { Acid } \\
\text { production }\end{array}$ & - & + & + & - & - \\
\hline 4. & $\begin{array}{l}\text { Pseudomonas } \\
\text { aeruoginosa }\end{array}$ & -ve Rod & Motile & - & - & - & + & + & - & $\begin{array}{l}\text { No change } \\
\text { of } \\
\text { production }\end{array}$ & + & + & + & - & - \\
\hline 5. & Streptococcus spp & $\begin{array}{l}+\mathrm{ve} \\
\text { cocci }\end{array}$ & $\begin{array}{l}\text { Non- } \\
\text { Motile }\end{array}$ & - & - & - & + & - & - & $\begin{array}{l}\text { Alkaline } \\
\text { production }\end{array}$ & - & - & + & + & + \\
\hline 6. & $\begin{array}{l}\text { Staphylococcus } \\
\text { spp }\end{array}$ & $\begin{array}{l}+\mathrm{ve} \\
\text { cocci }\end{array}$ & $\begin{array}{l}\text { Non- } \\
\text { Motile }\end{array}$ & - & - & - & + & + & + & $\begin{array}{l}\text { Alkaline } \\
\text { production }\end{array}$ & - & - & + & + & + \\
\hline 7. & Enterococcus spp & $\begin{array}{l}+\mathrm{ve} \\
\text { cocci }\end{array}$ & $\begin{array}{l}\text { Non- } \\
\text { Motile }\end{array}$ & - & - & + & - & - & - & $\begin{array}{l}\text { No } \\
\text { Acid,Gas } \\
\text { production }\end{array}$ & - & - & + & + & + \\
\hline 8. & Sulfobacillus spp & +ve Rod & \begin{tabular}{|l|} 
Non- \\
Motile \\
\end{tabular} & - & - & - & + & + & - & $\begin{array}{l}\text { Alkaline } \\
\text { production }\end{array}$ & + & - & + & + & + \\
\hline 9. & Escherichia coli & -ve Rod & Motile & - & - & + & - & - & - & $\begin{array}{l}\text { Acid,Gas } \\
\text { production }\end{array}$ & - & + & + & + & + \\
\hline 10. & Aeromonas spp & -ve Rod & Motile & + & - & + & + & + & - & $\begin{array}{l}\text { No acid } \\
\text { production }\end{array}$ & + & - & + & - & + \\
\hline 11. & $\begin{array}{l}\text { Brevibacterium } \\
\text { spp }\end{array}$ & +ve Rod & $\begin{array}{l}\text { Non- } \\
\text { Motile }\end{array}$ & - & + & - & + & + & - & $\begin{array}{l}\text { Alkaline } \\
\text { production }\end{array}$ & + & - & + & - & + \\
\hline 12. & Listeria spp & +ve Rod & Motile & - & + & + & - & + & - & $\begin{array}{l}\text { Acid } \\
\text { production }\end{array}$ & - & - & - & - & - \\
\hline 13. & Azotobacter spp & +ve Rod & $\begin{array}{l}\text { Non- } \\
\text { Motile }\end{array}$ & - & + & - & + & + & + & $\begin{array}{l}\text { Alkaline, } \\
\text { Acid } \\
\text { production }\end{array}$ & + & - & + & + & + \\
\hline 14. & Cellulomonas spp & +ve Rod & Motile & - & - & - & - & + & - & $\begin{array}{l}\text { No change } \\
\text { of } \\
\text { production }\end{array}$ & - & + & + & + & + \\
\hline 15. & $\begin{array}{l}\text { Corynebacterium } \\
\text { spp }\end{array}$ & +ve Rod & $\begin{array}{l}\text { Non- } \\
\text { Motile }\end{array}$ & - & + & - & - & + & + & $\begin{array}{l}\text { Acid } \\
\text { production }\end{array}$ & - & + & + & + & - \\
\hline 16. & Terrabacter spp & +ve Rod & $\begin{array}{l}\text { Non- } \\
\text { Motile }\end{array}$ & - & - & - & - & - & - & $\begin{array}{l}\text { No acid } \\
\text { production }\end{array}$ & + & + & - & - & - \\
\hline 17. & Aerococcus spp & $\begin{array}{l}+\mathrm{ve} \\
\text { cocci }\end{array}$ & $\begin{array}{l}\text { Non- } \\
\text { Motile }\end{array}$ & + & + & - & + & + & + & $\begin{array}{l}\text { Alkaline } \\
\text { production }\end{array}$ & + & - & + & + & + \\
\hline 18. & Klebsiella spp & -ve Rod & \begin{tabular}{|l|} 
Non- \\
Motile \\
\end{tabular} & - & - & + & + & + & + & $\begin{array}{l}\text { Acid,Gas } \\
\text { production }\end{array}$ & - & - & + & + & + \\
\hline 19. & Marinococcus spp & $\begin{array}{l}+\mathrm{ve} \\
\text { cocci }\end{array}$ & Motile & - & - & - & + & + & - & $\begin{array}{l}\text { Alkaline } \\
\text { production }\end{array}$ & + & + & - & + & + \\
\hline 20. & Sacchrococcus spp & $\begin{array}{l}+\mathrm{ve} \\
\text { cocci }\end{array}$ & $\begin{array}{l}\text { Non- } \\
\text { Motile }\end{array}$ & - & + & - & + & + & + & $\begin{array}{l}\text { Alkaline } \\
\text { production }\end{array}$ & + & - & + & + & + \\
\hline 21. & Enterobacter spp & -ve Rod & Motile & - & - & + & + & + & + & $\begin{array}{l}\text { Gas } \\
\text { production }\end{array}$ & - & + & + & + & - \\
\hline 22. & Thiobacillus spp & -ve Rod & \begin{tabular}{|l|} 
Non- \\
Motile
\end{tabular} & - & - & + & - & + & - & $\begin{array}{l}\text { Acis,Gas } \\
\text { production }\end{array}$ & + & - & + & + & + \\
\hline
\end{tabular}




\begin{tabular}{|r|l|l|l|l|l|l|l|l|l|l|l|l|l|l|l|l|}
\hline 23. & Planococcus spp & $\begin{array}{l}+ \text { ve } \\
\text { cocci }\end{array}$ & $\begin{array}{l}\text { Non- } \\
\text { Motile }\end{array}$ & + & + & - & + & + & - & $\begin{array}{l}\text { Alkaline } \\
\text { production }\end{array}$ & - & - & + & + & - \\
\hline 24. & Shigella spp & - ve Rod & $\begin{array}{l}\text { Non- } \\
\text { Motile }\end{array}$ & - & + & - & - & + & - & $\begin{array}{l}\text { H2S not } \\
\text { produced }\end{array}$ & - & - & & - & - & - \\
\hline 25. & Dermobacter spp & + ve Rod & $\begin{array}{l}\text { Non- } \\
\text { Motile }\end{array}$ & - & - & - & + & + & - & $\begin{array}{l}\text { Acid, } \\
\text { Alkaline } \\
\text { production }\end{array}$ & + & - & & + & + & + \\
\hline 26. & Salinococcus spp & + ve rod & Motile & - & - & - & - & + & - & $\begin{array}{l}\text { H2S S } \\
\text { production }\end{array}$ & + & + & + & - & - \\
\hline 27. & Deinococcus spp & + ve Rod & $\begin{array}{l}\text { Non- } \\
\text { Motile }\end{array}$ & + & - & - & + & + & - & $\begin{array}{l}\text { Alkaline } \\
\text { production }\end{array}$ & + & - & & + & + & + \\
\hline
\end{tabular}

Several studies suggested that soil microbial diversity had seasonal fluctuations (Lipson and Schmidt, 2004; Smit et al., 1997). Presence or absence of particular bacterial genera may depend on soil parameters, as observed by Alexander (Alexander, 1971). The Gram negative population and higher $\mathrm{O}_{2}$ level in sea water is conformed to previous reports by Gonzalez-Acosta and Ascencio et al. (2006).

Conversely the present study exposed higher Gram positive bacteria. The site of the present study soil supports for the presence of higher population of gram positive compare with gram negative, nitrifier, denitrifiers, phosphate solubilizer, sulphur oxidizers, for major biogeo-chemical cycles and also bioluminescent bacteria responsible for quorum sensing.

In conclusion, Mangrove soils endow with sanctuary and nurturing sites for many marine bacteria. Conservation strategies for mangroves should consider the ecosystem as a biological entity, which includes all the physical, chemical, and ecological processes that maintain productive mangroves. Outstanding to the presence of wealthy source of nutrients mangroves are called the homeland of microbes. Extensive exploration, identification, isolation and screening are suggested in search of new leads for microbial drugs.

\section{Acknowledgment}

The authors are thankful to Secretary and correspondent, the principal, A.V.V.M Sri Pushpam College (Autonomous), Poondi613503, Thanjavur for offering facilities to carry out this study.

\section{References}

Alexander, M. 1971. Microbial Ecol., Wiley, New York, USA.

Alongi, D.M., Christoffersen, P., Tirendi, F. 1993. The influence of forest type on microbial-nutrient relationships in tropical mangrove sediments, J. Exp. Mar. Biol. Ecol., 171: 201-223.

Bano, N., Nisa, M.U., Khan, N., Saleem, M., Harrison, P.J, Ahmed, S.I \& Azam, F. 1997. Significance of bacteria in the flux of organic matter in the tidal creeks of the mangrove ecosystem of the Indus river delta, Pakistan. Mar. Ecol. Prog. Ser., 157: 1-12.

Bernard, I., Schafer, H., Joux, F., Courties, C., Muyzer, G., Lebaron, P. 2000. Genetic diversity of total, active and culturable marine bacteria in coastal sea water. Aquat. Microb. Ecol., 23: 1-11.

Cappuccino, G.G., Sherman, N. 1999. Microbiology a Laboratory Manual Rockland Community College, Suffern: New York.

Fell, J.W., Master, I.M., Wiegert. R.G. 1984. Litter decomposition and nutrient 
enrichment. In: Snedaker SC, Snedaker JG (eds). The mangrove ecosystem: research methods. (Monograph on oceanographic methodology, no 8) UNESCO, Paris. 239-251.

Ganesh Babu, T., Nithyanand, P., Kannapiran, E., Veera Ravi, A., Karutha Pandian, S. 2004. Molecular identification of bacteria associated with the coral reef ecosystem of Gulf of Mannar Marine Biosphere Reserve using 16S rRNA sequences. In Proceedings of the National Seminar on New Frontiers in Marine Bioscience Research (Supplement) (eds Abidi, S. A. H. et al., National Institute of Ocean Technology, Chennai. pp. 47-53.

Gonzalez-Acosta, B., Bashan, Y., Hernandez- Saavedra, N.Y., Ascencio, F., Dela Cruz-Aguero, G. 2006. Seasonal seawater temperature as the major determinant for populations of culturable bacteria in the sediments of an intact mangrove in an arid region. FEMS. Microbiol. Ecol., 55: 311-321.

Holguin, G., Bashan, Y., Mendoza-Salgado, R.A., Amador, E., Toledo, G., Vazquez, P., Amador, A. 1999. La Microbiologia de los manglares, bosques en la frontera entre el mary la tierrra, Ciencia Desarrollo, 144: 26-35.

Kathiresan, K., Selvam, M.M. 2006. Evaluation of beneficial bacteria from mangrove. Soil. Bot. Mar., 49: 86-88.
Lipson, D.A., Schmidt, S.K. 2004. Seasonal changes in an alpine soil bacterial community in the Colorado Rocky Mountains. Appl. Environ. Microb., 70: 2867-2879.

Maria, G.L., Sridhar, K.R. 2002. Richness and diversity of filamentous fungi on woody litter of mangroves along the west coast of India. Curr. Sci., 83: 1573-1580.

Oliver, J.D. 1982. Taxonomy scheme for the identification of marine bacteria. Deep Sea Res., 29: 795-798.

Smit, E., Leeflang, P., Wernars, K. 1997. Detection of shifts in microbial community structure and diversity in soil caused by copper contamination using amplified ribosomal DNA restriction analysis. FEMS Microbiol Ecol., 23: 249-261.

Zhou, H.W., Guo, C.L., Wong, Y.S., Tam, N.F.Y. 2006. Genetic diversity of dioxygenase genes in polycyclic aromatic hydrocarbon-degrading bacteria isolated from mangrove sediments, FEMS. Microbiol. Lett., 262: 148-157.

Zobell, C.E. 1946. Marine Microbiology, Chronica Botanica Co, Waltham, Mass., USA. p. 240.

\section{How to cite this article:}

Saseeswari, A., G.Kanimozhi and Panneerselvam, A. 2016. Bacterial Diversity of Mangrove Soil in Karankadu from East Coast of Tamil Nadu, India. Int.J.Curr.Microbiol.App.Sci.5(4): 750-756. doi: http://dx.doi.org/10.20546/ijcmas.2016.504.086 